\title{
Evidence of the inhomogeneity of the stellar population in the differentially reddened globular cluster NGC 3201*
}

\author{
V. Kravtsov ${ }^{1,2}$, G. Alcaíno ${ }^{3}$, G. Marconi ${ }^{4}$, and F. Alvarado ${ }^{3}$ \\ 1 Instituto de Astronomía, Universidad Católica del Norte, Avenida Angamos 0610, Antofagasta, Chile \\ e-mail: vkravtsov@ucn.cl \\ 2 Sternberg Astronomical Institute, University Avenue 13, 119899 Moscow, Russia \\ 3 Isaac Newton Institute of Chile, Ministerio de Educación de Chile, Casilla 8-9, Correo 9, Santiago, Chile \\ e-mail: inewton@terra.cl, falvarad@eso.org \\ 4 ESO - European Southern Observatory, Alonso de Cordova 3107, Vitacura, Santiago, Chile \\ e-mail: gmarconi@eso.org
}

Received 26 November 2009 / Accepted 17 February 2010

\section{ABSTRACT}

\begin{abstract}
Aims. We report on evidence of the inhomogeneity (multiplicity) of the stellar population in the Galactic globular cluster (GC) NGC 3201, which is irregularly reddened across its face.

Methods. We carried out a more detailed and careful analysis of our recently published new multi-color photometry in a wide field of the cluster with particular emphasis on the $U$ band.

Results. Using the photometric data corrected for differential reddening, we found for the first time two key signs of the inhomogeneity in the cluster's stellar population and of its radial variation in the GC. These are (1) an obvious trend in the color-position diagram, based on the $(U-B)$ color-index, of red giant branch (RGB) stars, which shows that the farther from the cluster's center, the bluer on average the $(U-B)$ color of the stars is; and (2) the dependence of the radial distribution of sub-giant branch (SGB) stars in the cluster on their $U$ magnitude, where brighter stars are less centrally concentrated than their fainter counterparts at a confidence level varying between $99.2 \%$ and $99.9 \%$ depending on the color-index used to select the stars. The same effects were recently found by us in the GC NGC 1261. However, contrary to NGC 1261, we are not able to unambiguously suggest which of the sub-populations of $\mathrm{SGB} / \mathrm{RGB}$ stars can be the progenitor of blue and red horizontal branch stars of the cluster. Apart from M4, NGC 3201 is another GC very probably with an inhomogeneous stellar population, which has essentially lower mass than the most massive Galactic GCs where multiple stellar populations were unambiguously detected for the first time.
\end{abstract}

Key words. globular clusters: general - globular clusters: individual: NGC 3201

\section{Introduction}

The southern Galactic globular cluster (GC) NGC 3201, known not only by its peculiar kinematic characteristics but also by irregular differential reddening across its face, was the subject of our recent study (Kravtsov et al. 2009) based on a new multicolor photometry in a $14^{\prime} \times 14^{\prime}$ field of the GC. In that study, where we primarily dealt with some aspects of the properties and characteristics of the cluster stellar population, we also allowed a possible spread in the population, but did not examine it. In a later more detailed analysis of the same data, we were able to find not only apparent manifestations, but also stronger and more objective evidence of the inhomogeneity in the stellar population and of its radial variation in the cluster. The present letter reports on these findings in detail. The obtained results contribute more to our past (Alcaíno et al. 1999) and recent (Kravtsov et al. 2010) studies of the inhomogeneity (multiplicity) of the stellar populations in the populous Large Magellanic Cloud (LMC) cluster NGC 1978 and Galactic GC NGC 1261, respectively and to the rapidly growing body of photometric and spectroscopic evidence about multiple stellar populations in both Magellanic Clouds star clusters (e.g., Mackey et al. 2008; Milone et al. 2009a; and

* Based on observations with the $1.3 \mathrm{~m}$ Warsaw telescope at Las Campanas Observatory. references therein) and Galactic GCs (some relevant publications are referred to elsewhere in the paper).

\section{The used photometric data and the corrections applied to them}

In the present study, we are relying on our recent multi-color photometry in $U B V I$ of more than 12000 stars in a $14^{\prime} \times 14^{\prime}$ cluster field, reaching below the turnoff point in all passbands. For a description of the photometric data used here (and publicly available in electronic form) and of the typical rms errors in each passband, see Kravtsov et al. (2009). In the same paper, we also described in detail our study of the variation of reddening in the observed cluster field and listed the estimated corrections corresponding to areas with conditionally defined grades of reddening. These corrections were estimated separately for the $U$ and $V$ magnitudes, as well as for the $(B-V),(V-I)$, and $(B-I)$ colors, and typical uncertainties were quoted. As for the corrections for the $(U-B)$, they were supplementary calculated in the present study from those originally obtained for the $(B-V)$ color by applying the following relation between the color-excesses in both colors: $E_{U-B}=0.72 E_{B-V}$. In the analysis presented below, we used photometric data and the cluster's CMDs corrected for differential reddening. 
In addition to its variable reddening, the cluster field is also populated by the large number of field stars, which are fairly numerous in the region of the lower red giant branch (RGB) and sub-giant branch (SGB). A decontamination procedure was applied to the photometry used. For more detail, interested readers are referred to our original paper on photometry of NGC 3201.

\section{The sub-giant branch in the $U$ magnitude}

In two rows of panels of Fig. 1, we demonstrate the apparent radial variation of the $U$-level of the SGB of NGC 3201 in the $U$-based CMDs with different color-indexes. The CMDs are corrected for differential reddening and decontaminated of the majority of field stars in the region of the lower RGB and of the SGB. The CMDs shown in the upper and lower panels correspond to the inner $(1 ! 35<R<2 ! 70)$ and the outer $\left(R>3{ }^{\prime} 4\right)$ regions of the cluster, respectively. One can see that at least the $U$-level and perhaps the slope of the SGB vary with the distance from the center of NGC 3201, the SGB being systematically brighter (and perhaps steeper) in the outer part of the cluster. The dashed horizontal line is drawn at the same $U$ magnitude in both rows of panels as a reference line to facilitate a comparison of the SGB level in the CMDs of the two parts in the cluster.

The qualitatively demonstrated radial variations of the distribution of stars on the SGB in the $U$-based CMDs are supported by more objective indicators based on quantitative estimates. They were obtained in the following way. We first isolated a sample of stars most probably belonging to the SGB. The $U$-based cluster CMD with the $(B-I)$ color-index was initially used to this goal. This color-index provides the largest separation between the turnoff (TO) point and the lower RGB, as compared with other available color-indices corrected for differential reddening. The central part of the cluster was not excluded from the analysis, thanks to only a weak crowding effect in it. Given a slight apparent slope of the SGB in the $U-(B-I)$ CMD, it was taken into account in isolating SGB stars. They were selected in the color range $\Delta(B-I)=0.25(1.75<B-I<2.00)$ and the magnitude range $\Delta U=0.4$ mag between the accepted upper and lower borders of the branch, defined by two envelope lines, $U=-0.194(B-I)+18.75$ and $U=-0.194(B-I)+19.15$. The total number of the selected stars is 415 . We then divided the obtained sample of the SGB stars by three sub-samples: (1) a sub-sample of 115 brightest SGB stars in the magnitude range $\Delta U=0.15$ mag; (2) a sub-sample of 178 faintest SGB stars also confined in the magnitude range $\Delta U=0.15 \mathrm{mag}$; (3) a sub-sample of $122 \mathrm{SGB}$ stars falling in the magnitude range $\Delta U=0.10 \mathrm{mag}$ in between the two extreme ranges. Then we also similarly selected SGB stars by relying independently on each of the two additional color-indices, $(B-V)$ and $(V-I)$, in their ranges $\Delta(B-V)=0.12(0.75<B-V<0.87)$ and $\Delta(V-I)=0.13(1.00<V-I<1.13)$, respectively. A total magnitude range of the SGB, $\Delta U=0.4$ mag and a division of it into three parts yields the same as in the $U-(B-I)$ CMD, with the same $U$ magnitudes defining the boundaries of these parts at the extremes of each color range as was accepted for the SGB in the three color-indices. Note that although the color-indices under consideration are not independent (i.e., each of them is a combination of two other color-indices), the corrections for differential reddening were originally made independently in each of them. The number of stars falling in the fainter, intermediate, and brighter boxes are 188, 130, and 117 respectively in the $U-(B-V)$ CMD. In turn, the boxes of the same sequence of the brightness levels in the $U-(V-I) \mathrm{CMD}$ contain 165, 125, and 114 stars, respectively. For comparison

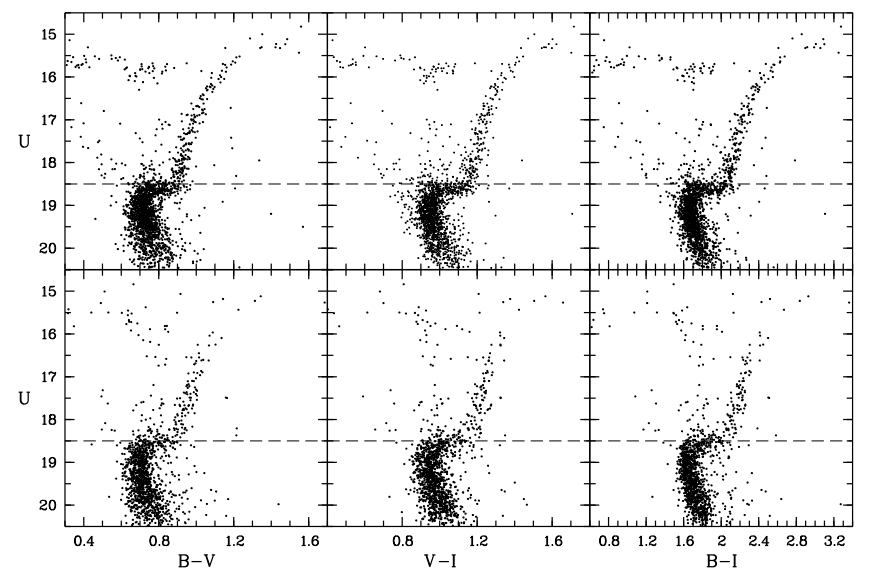

Fig. 1. The $U$-based CMDs with different color-indexes, corrected for differential reddening and (partially) rid of field stars, demonstrating the systematic variation of the position of the SGB in the $U$ with radial distance from the center of NGC 3201. Upper and lower panels show the CMDs of inner $\left(1.35<R<2\right.$ '.70) and outer $\left(R>3{ }^{\prime} \cdot 40\right)$ regions of the cluster, respectively. The dashed horizontal line is given as a reference to facilitating a comparison of the SGB in the CMDs.

purposes, we also isolated SGB stars in the $V$ based CMD, namely $V-(V-I)$. The SGB is apparently slightly narrower in the $V$ magnitude, i.e. $\Delta V=0.35 \mathrm{mag}$ as compared to $\Delta U=$ $0.4 \mathrm{mag}$. The color range is the same as in the $U-(V-I)$ CMD. The accepted upper and lower borders of the SGB in the $V-(V-I)$ were defined by two envelope lines, $V=-1.923(V-$ $I)+19.87$ and $V=-1.923(V-I)+19.52$. Taken the narrower SGB in the $V$ magnitude into account, we selected sub-samples of the brightest and faintest SGB stars in slightly reduced magnitude ranges than in the $U$ magnitude, namely $\Delta V=0.13 \mathrm{mag}$ (98 stars) and $\Delta V=0.12 \mathrm{mag}$ (136 stars), respectively. The magnitude range of the box containing the sub-sample of SGB stars of intermediate brightness (131 items) was kept unchanged.

The selection boxes are drawn by the solid lines in the CMDs in the upper panel of Fig. 2, where the corresponding selected stars of the three sub-samples with progressively increasing brightness are denoted by red, black, and blue filled circles. The lower panels of Fig. 2 show the cumulative radial distributions of these sub-samples of SGB stars. As for the stars isolated in the three $U$ based CMDs, one can see that the faintest sub-giants denoted by (red) filled triangles are more centrally concentrated than their counterparts of intermediate brightness in the $U$ (black filled circles), and obviously yet more centrally concentrated than the brightest SGB stars (blue filled squares), irrespective of the color-index used for the selection of the SGB stars. This apparent difference between the distributions is supported by a quantitative estimate based on a KolmogorovSmirnov test: the difference between the radial distributions of the brightest and faintest SGB stars is statistically significant at $99.8 \%, 99.2 \%$, and $99.9 \%$ confidence levels for the selection made in the $U-(B-I), U-(B-V)$, and $U-(V-I)$ CMDs, respectively. However, the radial distributions of the subsamples of SGB stars with different brightness in the $V$ magnitude are apparently in contrast to those differing by the $U$ magnitude. In particular, the brightest SGB stars in the $V$ passband are even somewhat more centrally concentrated in the central part of NGC 3201 than their faintest counterparts. Moreover, the radial distributions of the two sub-samples of SGB stars are different at $92.1 \%$ confidence level. Such a difference is, strictly speaking, 

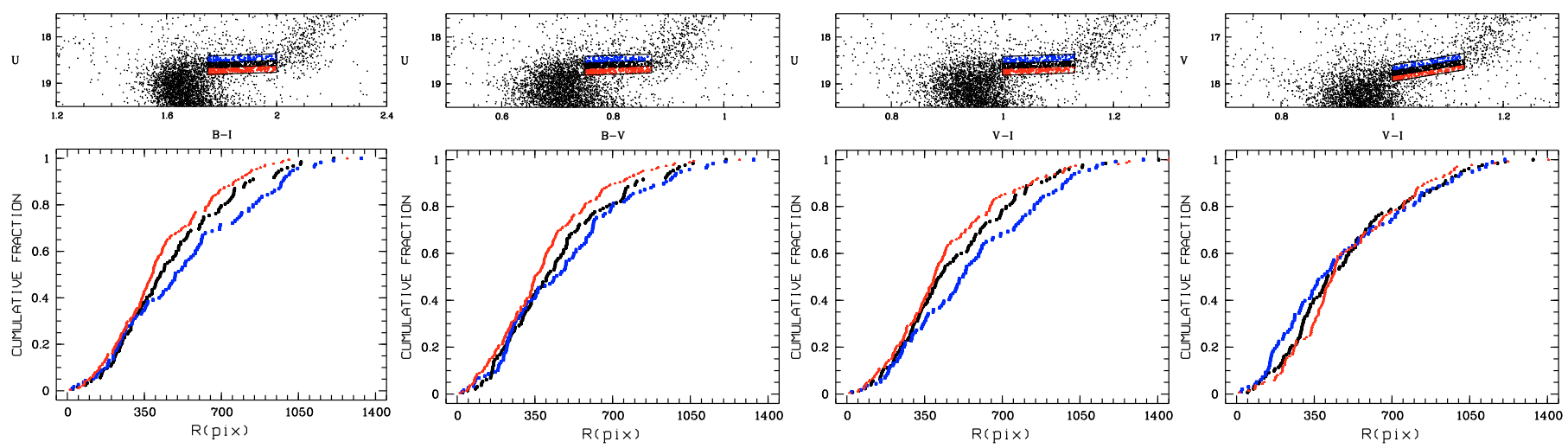

Fig. 2. Upper panels show three sub-samples of the SGB stars isolated in three magnitude ranges: (1) in the $U$-based CMDs of NGC 3201 with different color-indices, and additionally; (2) in the $V-(V-I) \mathrm{CMD}$, see text for details. All the CMDs are corrected for differential reddening. Lower panels: a comparison of the cumulative radial distributions of the three sub-samples of SGB stars; red filled triangles, black filled circles, and blue filled squares denote the sub-samples of the SGB stars with progressively increasing brightness in the given passbands. In all the panels, the three sub-samples are shown with symbols of the same color.

statistically insignificant, because the estimated confidence level is less than $95.0 \%$.

The above result may imply the following. Even if the dependence of the radial distribution of SGB stars on their brightness in the $V$ magnitude (at a given color) really exists and has the same meaning as the dependence in the $U$ magnitude, it is presumably less obvious, so that the errors of the applied corrections for differential reddening combined with the errors of the photometry itself are able to reduce or even distort the dependence.

The high level of statistical significance of the dependence of the SGB stars' radial distribution in NGC 3201 on their $U$ magnitude is strong evidence of the inhomogeneity ("multiplicity") of the cluster stellar population. This high level was also found by us in another Galactic GC, NGC 1261. Here we note again that the inhomogeneity of the population of SGB stars resulting from the revealed dependence in both GCs resembles a combination of two effects: (1) the split of the SGB into two components revealed by Milone et al. (2008) in the GC NGC 1851; and (2) the different radial distribution of stars belonging to the brighter and fainter components of the SGB, found by Zoccali et al. 2009) in the same GC, i.e. the brighter SGB stars were found to be less centrally concentrated and to extend to much larger radial distances in NGC 1851. Note however that Milone et al. (2009b) do not support this finding.

As for the revealed differences regarding SGB stars in NGC 3201, one cannot draw any definite conclusion about whether or not they are of a discrete or a continuous character.

\section{The red giant branch and its color-position diagram}

The obtained evidence of the probable inhomogeneity of SGB stars in NGC 3201 is reinforced by the inhomogeneity of RGB stars and its dependence on the radial distance in the cluster. For our analysis of the RGB of NGC 3201, we used a ready-made sample of the most probable RGB stars. Their selection was done previously and is described in detail in Kravtsov et al. (2009). Based on this sample we found as in the RGB of NGC 1261 a systematically different location in the $U-(U-B)$ diagram of RGB stars situated at different radial distances from the cluster center, where the portion of stars bluer in the $(U-B)$ systematically increased towards the cluster outskirts. In Fig. 3

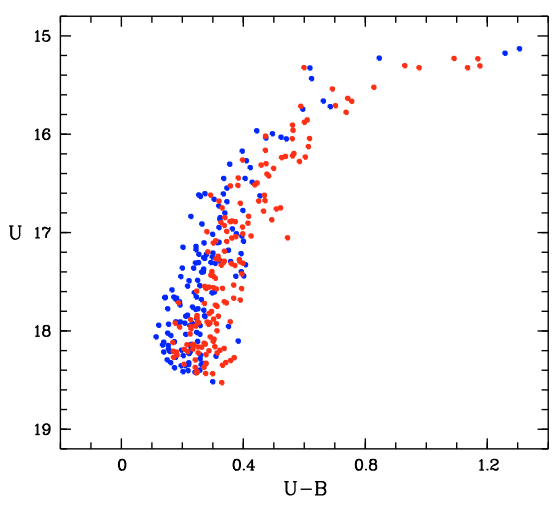

Fig. 3. Comparison of the location in the $U-(U-B) \mathrm{CMD}$ of RGB stars from the inner (red dots, 1 ' $35<R<2$ '.70) and outer (blue dots, $3.4<$ $R<6.3$ ) regions of NGC 3201 .

we compare in the $U-(U-B)$ diagram the position of two sub-samples of RGB stars belonging to two areas at different mean radial distances from the cluster center, namely in the radial ranges $1.35<R<2$ '.70 and 3'.4<R<6.3. It is evident that RGB stars in the outer region (blue dots) are systematically bluer than in the inner one.

To secure more convincing evidence and more detailed behavior of the suggested dependence of the $(U-B)$ color of RGB stars on their radial distance, R, from the center of NGC 3201, we obtained the so-called color-position diagram (CPD) of the RGB. Note that this diagram was used for the first time in our study of the stellar population in the LMC populous star cluster NGC 1978 (Alcaíno et al. 1999). First of all, we linearized the RGB in the $U-(U-B)$ plane by relying on the readymade sample of the most probable RGB stars. Since the brightest part of the RGB, formed by a dozen of stars with $U<15.3$, is quasi-horizontal in the $U$-based CMDs, we rejected these stars. We fitted the mean locus of the RGB with a polynomial and subtracted for each star the color of the mean locus at its luminosity level from the star's color-index. We left nearly all stars of the initial sample. Only a handful of stars with deviations, $\delta(U-B)$, from the mean locus exceeding \pm 0.15 mag in the total range of the RGB in the $U$ magnitude were rejected. Note that the number of rejected stars is so small that this is rather a formal procedure with negligible impact. The linearized RGB is shown in the $U-\delta(U-B)$ plane in the upper panel of 


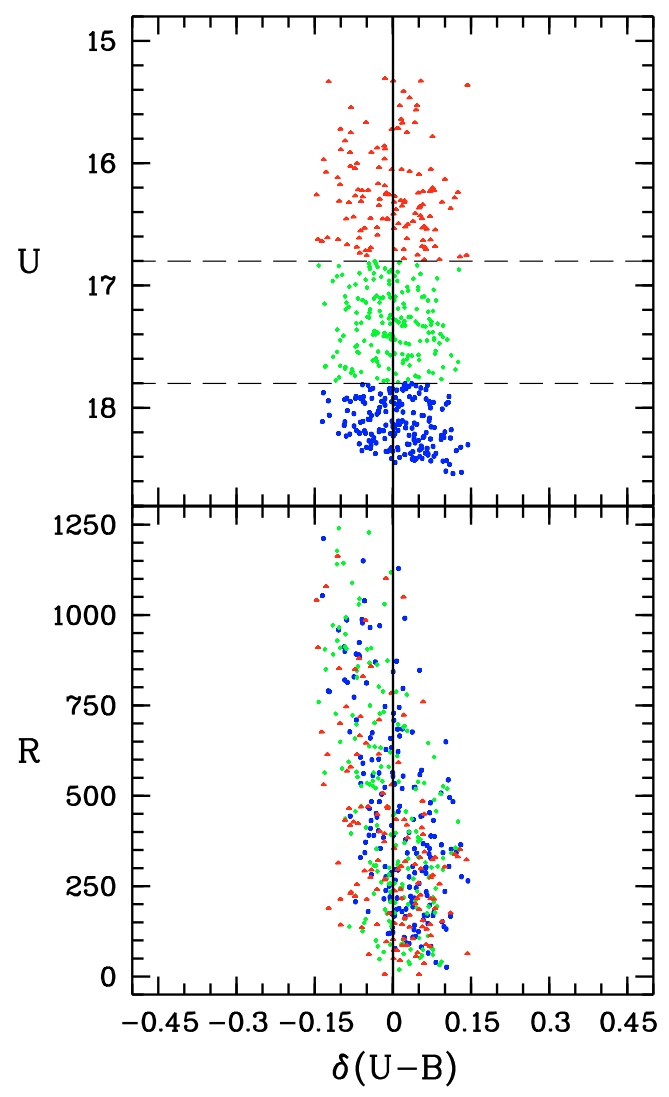

Fig. 4. The trend of the $(U-B)$ color of RGB stars with their radial distance from the center of NGC 3201. Upper panel: the linearized RGB for the color $(U-B)$ corrected for differential reddening. A dozen of the brightest stars of the RGB $(U<15.3)$ were rejected. For demonstration purposes, the RGB was arbitrarily divided into three magnitude intervals of comparable samples of stars, marked by dashed lines, where stars are denoted by different symbols. The lower panel shows the color-position diagram of the same RGB stars.

Fig. 4. With the purpose explained below, the linearized RGB was arbitrarily divided by three magnitude ranges (marked by the dashed lines) with comparable samples of stars, namely: 189 faint stars with $U>17.8 ; 185$ stars with intermediate brightness, $16.8<U<17.8 ; 113$ brighter stars with $U<16.8$. The final step is plotting the dependence between the deviations $\delta(U-B)$ of the RGB stars and their radial distances from the center of NGC 3201.

The obtained CPD shown in the lower panel of Fig. 4 is not only another representation of the demonstrated photometric inhomogeneity of RGB stars in NGC 3201, but also a dependence containing additional information. It is now evident beyond doubt that the $(U-B)$ color does get bluer, i.e. the $\delta(U-B)$ becomes more negative with increasing radial distance from the cluster center. It is also obvious that all the three sub-samples of RGB stars follow the same trend. Note yet that the CPD assumes the trend apparently reveals itself beginning at $R \approx 350-400$ pixels $(2.35-2.70)$ and there is no obvious trend within this radial distance, where the bulk of stars have positive deviations $\delta(U-B)$. Interestingly enough, this radius is virtually equal to the cluster half-mass radius, $R_{\mathrm{h}}=2$ '.68 (Harris 1996). In this connection it should be also noted that the difference in radial distribution among SGB stars arises approximately at the same radius.

Concerning the physical reasons that can probably be responsible for the revealed inhomogeneity, we refer to recently obtained results by Marino et al. (2008) on stellar population in GC M4. Based on spectroscopy of a sample of 105 stars in this $\mathrm{GC}$ they found a dichotomy in $\mathrm{Na}$ abundance and argued that it must be associated with a $\mathrm{CN}$ bimodality. From photometry of the same stars Marino et al. (2008) showed that the CN-weak red giants with a lower content of $\mathrm{Na}$ are on average systematically bluer, by $\Delta(U-B)=0.17$ in the $U-(U-B) \mathrm{CMD}$, than their $\mathrm{CN}$-strong counterparts with a higher content of $\mathrm{Na}$. This photometric effect is very similar to that revealed by us in NGC 3201 and in NGC 1261. From the CPD we evaluate the mean separation in the $(U-B)$ color between "blue" and "red" RGB stars, assuming this bi-modality in their distribution in the $(U-B)$ color. It is apparently around $\Delta(U-B) \sim 0.12$, which is many times larger than the possible error in the $(U-B)$ color (a few hundredth of magnitudes in the worse case) caused by the uncertainty in local fluctuations of reddening in the cluster field under consideration. It agrees well with our estimate made for NGC 1261 and is on the same order of magnitude as the abovementioned difference between the "redder" and "bluer" giants in M4. A tentative first explanation is to accept the same reason behind the segregation of RGB stars in the color $(U-B)$ in NGC 3201 as in M4.

In NGC 3201, other than in NGC 1261, we are not able to suggest an unambiguous association between the discussed subpopulations of SGB/RGB stars and those belonging to the blue and red horizontal branch (BHB, RHB).

\section{Conclusions}

Based on a more detailed analysis of a new multi-color photometry in an extended field of the differentially reddened GC NGC 3201, we found the following signs of the inhomogeneity (multiplicity) of the cluster's stellar population. First, there is an obvious dependence of the radial distribution of SGB stars in the cluster on their $U$ magnitude: brighter stars are less centrally concentrated than their fainter counterparts at a confidence level fluctuating above $99.2 \%$ in relation to the colorindex of CMD relied on to isolate SGB stars. Second, RGB stars exhibit a systematically different location in both the $U-(U-B)$ CMD and the $R-\delta(U-B)$ color-position diagram at different radial distances from the cluster center: the proportion of stars bluer in the $(U-B)$ increases towards the cluster outskirts. We note (1) the same kind of photometric inhomogeneity of RGB and SGB stars in NGC 3201 and in another Galactic GC, NGC 1261 (Kravtsov et al. 2010); and also (2) a very similar radial trend in both GCs.

Finally, it is worth mentioning that NGC 3201 after M4, is the second non-massive Galactic GC that very probably has an inhomogeneous stellar population.

Acknowledgements. We thank the anonymous referee for useful comments that improved the manuscript.

\section{References}

Alcaíno, G., Liller, W., Alvarado, F., et al. 1999, A\&AS, 135, 103 Harris, W. E. 1996, AJ, 112, 1487

Kravtsov, V., Alcaíno, G., Marconi, G., et al. 2009, A\&A, 497, 371 Kravtsov, V., Alcaíno, G., Marconi, G., et al. 2010, A\&A, accepted Mackey, A. D., Broby Nielsen, P., Ferguson, A. M. N., et al. 2008, ApJ, 681, L17 Marino, A. F., Villanova, S., Piotto, G., et al. 2008, A\&A, 490, 625 Milone, A. P., Bedin, L. R., Piotto, G., et al. 2008, ApJ, 673, 241 Milone, A. P., Bedin, L. R., Piotto, G., et al. 2009a, A\&A, 497, 755 Milone, A. P., Stetson, P. B., Piotto, G., et al. 2009b, A\&A, 503, 755 Zinn, R., \& West, M. J. 1984, ApJS, 55, 45

Zoccali, M., Piotto, G., Zaggia, S. R., et al. 1998, A\&A, 331, 541 Zoccali, M., Pancino, E., Catelan, M., et al. 2009, ApJ, 697, L22 